triglycerides $=172 \mathrm{mg} / \mathrm{dl}$ ) and indications of treatment with Medrol $2 \mathrm{mg} / \mathrm{kg}$ with gradual reduction of dosage and Aspirin $4 \mathrm{mg} / \mathrm{kg}$.

After about 1 month he returns for revaluation, showing good general condition, upward weight curve, biological samples within normal limits.

Ultrasound cardiological reassessment and CT angiography further reveal significant aneurysmal dilatation of the left coronary artery. Anticoagulant treatment was completed with enoxaparin.

Conclusions KAWASAKI disease associated with COVID 19, may present an unfavorable outcome with lack of response to the initial immunoglobulin treatment and evolution to coronary aneurysm.

\section{CARDIAC INVOLVEMENT AND SHORT-TERM OUTCOMES OF SARS COV 2 INFECTED PATIENTS WITH PEDIATRIC MULTI-SYSTEMIC INFLAMMATORY SYNDROME AT AN EMERGENCY CLINICAL SETTING IN BUCHAREST}

Ana Maria Jurca*, Ioana Bejan, Evelina Aloma Cotora, Carmen-Cristina Olteanu. Grigore Alexandrescu Emergency Hospital for Children

\subsection{6/archdischild-2021-europaediatrics.186}

Pediatric multi-systemic inflammatory syndrome (PIMS) has gained attention throughout the medical world due to the ongoing SARS COV 2 pandemic. Being a systemic inflammatory response, cardiovascular complications are no exception, thus awareness of such conditions must be raised, as well as of prompt and accurate diagnosis and treatment. Attention is particularly brought to PIMS due to the fact that symptoms of it overlap with Kawasaki disease and toxic shock syndrome. The aim of this paper is to present cardiac findings and short-term outcomes in children with PIMS admitted to one of the main pediatric emergency health care units in Romania.

This current paper draws data from a single center, and is an observational, prospective study. The number of patients that were included in this study is 26 , with age range between 1 month and 17 years, hospitalized between December 2020 and April 2021 in the 'Grigore Alexandrescu' Emergency Hospital for Children, Bucharest. The main criterion for inclusion was PIMS as defined by CDC/WHO case definition. Out of all symptoms, persistent fever and gastrointestinal conditions were de most frequent ones ( $88 \%$ and $69 \%$, respectively).

Cardiac involvement was found in 11 patients (42\%), being represented by: left ventricular dysfunction (26\%), coronary artery abnormalities (15\%), atrioventricular valve regurgitation (30\%), with only one patient showing pericardial effusion and one an ECG anomaly. Improvement of initial clinical symptoms was paralleled by alleviation of cardiac symptomatology, as well as normalization of cardiac and inflammatory laboratory findings.

Our study shows that cardiac involvement is frequent in pediatric population with systemic inflammatory syndrome and we believe that PIMS in SARS COV 2 infected patients should be thoroughly screened and treated by a multi-disciplinary team. 24 out of 26 patients were fully recovered and discharged without sequelae, but two patients with persistent coronary artery aneurysmal findings.

\section{7 KAWASAKI DISEASE TREATMENT EXPERIENCE IN UHC SESTRE MILOSRDNICE}

${ }^{1}$ Suzana Bitanga*, ${ }^{1}$ Matej Katavić, ${ }^{2}$ Ingrid Sitaš, ${ }^{1}$ Nikola Krmek, ${ }^{1}$ Hrvoje Kniewald. ${ }^{1}$ Pediatric Cardiology Section, Pediatric Department, UHC Sestre milosrdnice; ${ }^{2}$ Diagnostic and General Pediatric Section, Pediatric Department, UHC Sestre milosrdnice

\subsection{6/archdischild-2021-europaediatrics.187}

Introduction Kawasaki disease (KD) is one of the most common systemic vasculitis predominantly involving coronary arteries with unknown etiology. It is generally assumed as inflammation of blood vessels predominantly affecting the cardiovascular system and possibility of developing long-term complications (primarily aneurysms and coronary artery thrombosis). It most often occurs in infants and preschool children, especially boys with much higher incidence of complications in younger children, notably if the disease remains unrecognized and untreated. In the period from 2012 to 2019, we hospitalized and treated five children with Kawasaki disease, three girls and two boys aged four to seven years. The disease was diagnosed in all patients based on clinical criteria. Echocardiographical changes in the heart and coronary arteries were registered (primarily pericardial effusion, mitral regurgitation and perivascular hyperechogenicity in the coronary arteries of varying intensity, in one child ectasia of the initial part of the LCA was noted). After IVIG therapy, all patients became afebrile within 24 hours, with no long-term cardiovascular complications.

Case Report S.K., a 4-year-old girl was treated as an outpatient with peroral penicillin for suspected scarlet fever. On the fourth day of the disease abnormal urine testing was noted and cephalosporin antibiotic introduced into the therapy. On the fifth day of the illness, she was admitted to the Gastroenterology Section of our Department due to suspected pancreatitis with hepatic lesion and acholic stools. Abdominal ultrasound revealed gallbladder hydrops. The day after that conjunctival hyperemia was noted. Because of the suspected $\mathrm{KD}$ echocardiography was performed with mild mitral regurgitation, enlargement of the ostium LCA (+3 SD for body surface) and mild pericardial effusion registered.

A.S., a 5-years-old boy was admitted to the Respiratory Section due to generalized urticaria with perirobital edema and bloated hands. Initially treated with antihistamines, he received a single dose of parenteral corticosteroid. With the appearance of scarlatiniformn rash and raspberry tongue, scarlet fever was suspected and peroral penicillin introduced. While being unresponsive to antimicrobial therapy, and with the appearance of conjunctival hyperemia, swelling of the feet and elevated liver enzymes, Kawasaki's disease was suspected. Echocardiographically pericardial effusion and negative $\mathrm{T}$ waves in the ECG were found.

D.M., a 5 -year-old boy. His disease began with high fever, sore throat, dysphagia, neck pain and neck stiffness. On the third day of the disease visible swelling on the right side of the neck $(3 \times 5 \mathrm{~cm})$ was noted with erythema of the feet, palms and axillae. He was hospitalized with aim to treat parapharyngeal abscess as a complication after unsuccessful penicillin therapy for Streptococcal pharyngitis and scarlet fever. After the initial work-up (ENT examination, MSCT of the neck), surgical removal of the lymph node was performed and ceftriaxone introduced. On the fourth day of the disease, edema 